\title{
Avaliação e monitoramento dos níveis de contaminação por resíduos de agrotóxicos em alimentos de origem vegetal comercializados no Estado de Minas Gerais, Brasil
}

\author{
Assessment and monitoring of contamination levels by pesticide residues in foods of plant origin \\ marketed in the State of Minas Gerais, Brazil \\ Evaluación y seguimiento de niveles de contaminación por residuos de plaguicidas en alimentos de \\ origen vegetal comercializados en el Estado de Minas Gerais, Brasil
}

\section{Resumo}

Nos últimos anos, tem sido crescente a preocupação dos consumidores com a presença de resíduos de agrotóxicos em alimentos, principalmente em vegetais frescos. Este estudo teve como objetivo descrever e avaliar os resultados da análise de resíduos de agrotóxicos em alimentos vegetais coletados pelo Programa de Análise de Resíduos de Agrotóxicos em Alimentos (PARA) no estado de Minas Gerais, Brasil. O método consistiu na análise documental e descritiva dos laudos de análises dos alimentos coletados no comércio varejista, entre os anos de 2013 e 2017 . Os resultados revelaram que $62,3 \%$ das amostras analisadas apresentaram resíduos de agrotóxicos, destas 22,6\% apresentavam resultados insatisfatórios perante a legislação. As culturas alimentares mais reprovadas foram, respectivamente: abobrinha, pimentão, goiaba, uva, morango e pepino. Considerando todas as amostras analisadas, $21,6 \%$ foram rejeitadas por apresentarem princípio ativo não autorizado, 3\% por conterem agrotóxicos acima dos limites máximos de resíduos e $2 \%$ foram reprovadas em ambos os parâmetros. Além disso, 5,8\% das amostras insatisfatórias apresentaram agrotóxicos cujo uso é proibido no país. O presente estudo permitiu verificar a presença de resultados insatisfatórios para resíduos de agrotóxicos em expressiva parcela de alimentos vegetais comercializados em Minas Gerais, o que reforça a necessidade da implementação de ações mais efetivas de controle e mitigação dos riscos frente a contaminação de alimentos por agrotóxicos.

Palavras-chave: Agroquímicos; Vigilância sanitária; Controle de qualidade; Frutas e hortaliças; Segurança alimentar.

\begin{abstract}
In recent years, there has been a growing concern among consumers about the presence of pesticide residues in food, especially in fresh vegetables. This study aimed to describe and evaluate the results of the analysis of pesticide residues in plant foods collected by the Program for the Analysis of Pesticide Residues in Food (PARA) in the state of Minas Gerais, Brazil. The method consisted of documentary and descriptive analysis of the analysis reports of the food collected in the retail trade, between the years 2013 and 2017. The results revealed that $62.3 \%$ of the analyzed samples presented pesticide residues, of these $22.6 \%$ presented results unsatisfactory under the legislation. The most disapproved food crops were, respectively: zucchini, peppers, guava, grapes, strawberries and cucumbers. Considering all the samples analyzed, $21.6 \%$ were rejected for presenting an unauthorized active ingredient, $3 \%$ for containing pesticides above the maximum residue limits and $2 \%$ were rejected in both parameters. In addition, $5.8 \%$
\end{abstract}


of unsatisfactory samples presented pesticides whose use is prohibited in the country. The present study allowed to verify the presence of unsatisfactory results for pesticide residues in a significant portion of plant foods sold in Minas Gerais, which reinforces the need to implement more effective actions to control and mitigate risks in the face of food contamination by pesticides.

Keywords: Agrochemicals; Health surveillance; Quality control; Fruits and vegetables; Food security.

\section{Resumen}

En los últimos años, ha habido una creciente preocupación entre los consumidores por la presencia de residuos de plaguicidas en los alimentos, especialmente en las verduras frescas. Este estudio tuvo como objetivo describir y evaluar los resultados del análisis de residuos de plaguicidas en alimentos vegetales recolectados por el Programa de Análisis de Residuos de Plaguicidas en Alimentos (PARA) en el estado de Minas Gerais, Brasil. El método consistió en el análisis documental y descriptivo de los informes de análisis de los alimentos recolectados en el comercio minorista, entre los años 2013 y 2017. Los resultados revelaron que el 62,3\% de las muestras analizadas presentaron residuos de plaguicidas, de estas $22,6 \%$ presentaron resultados insatisfactorios bajo la legislación. Los cultivos alimentarios más desaprobados fueron, respectivamente: calabacín, pimiento, guayaba, uvas, fresas y pepinos. Considerando todas las muestras analizadas, el $21,6 \%$ fueron rechazadas por presentar un ingrediente activo no autorizado, el $3 \%$ por contener plaguicidas por encima de los límites máximos de residuos y el $2 \%$ fueron rechazados en ambos parámetros. Además, el 5,8\% de las muestras insatisfactorias presentaron plaguicidas cuyo uso está prohibido en el país. El presente estudio permitió verificar la presencia de resultados insatisfactorios para residuos de plaguicidas en una porción significativa de alimentos vegetales vendidos en Minas Gerais, lo que refuerza la necesidad de implementar acciones más efectivas para controlar y mitigar riesgos ante la contaminación de alimentos por plaguicidas.

Palabras clave: Agroquímicos; Vigilancia sanitaria; Control de calidad; Frutas y vegetales; Seguridad alimentaria.

\section{Introdução}

De acordo com o último Censo Agropecuário realizado em 2017 no Brasil, o estado de Minas Gerais possuía 607,6 mil estabelecimentos agropecuários, uma área plantada de 38,2 milhões de hectares e era responsável pelo emprego de 1,8 milhão de trabalhadores rurais. Entre os principais alimentos produzidos no estado podem ser citados café arábica, alho, feijão, abóbora, morango, abacaxi, banana e batata. Pelos dados do Censo, cerca de 1/3 dos agricultores do estado relataram o uso de agrotóxicos na produção de alimentos (IBGE, 2019).

Em geral, o modelo de desenvolvimento agrícola no estado de Minas Gerais, assim como no restante do país, tem sido impulsionado pelo agronegócio, cuja produção agrícola é centrada na monocultura, no plantio de grandes latifúndios, na mecanização em larga escala, na precariedade das relações de trabalho e na elevação dos riscos ambientais (Balsan, 2006; Neto, Lacaz \& Pignati, 2014). Para alguns autores, esse modelo resultou em uma agricultura cada vez mais dependente do uso de agrotóxicos (Pelaez et al., 2015; Bombardi, 2017), de forma que o ritmo de evolução do mercado de agrotóxicos brasileiro tem apresentado crescimento superior ao do mercado mundial, o que tornou o país um dos maiores mercados consumidores de agrotóxicos do mundo (Anvisa, 2012; Santos \& Glass, 2018; IPEA, 2019).

Para Schreinemachers e Tipraqsa (2012), um aumento de 1\% na produção agrícola por hectare está associado a um aumento de 1,8\% no uso de agrotóxicos na agricultura, sendo que este aumento é ainda maior em países subdesenvolvidos, cujas as substâncias utilizadas para o controle químico ainda são fracamente reguladas.

A recente discussão sobre a reavaliação e flexibilização do processo de registro de agrotóxicos no Brasil, impulsionada por interesses da indústria e pela influência do setor ruralista (Friedrich, Souza \& Carneiro, 2018), resultou na publicação de um novo marco regulatório no país, contendo novos critérios de avaliação e classificação toxicológica referentes ao registro e a autorização do uso agrotóxicos (Anvisa, 2019a; Anvisa, 2019b).

De acordo com os dados disponíveis no sistema de registro de agrotóxicos fitossanitários, no período de 2016 a 2020 o Brasil autorizou o uso de 2.012 novos ingredientes ativos de agrotóxicos, um aumento de 146,9\% em relação aos cinco anos anteriores, quando foram liberados 815 ativos no país (Brasil, 2021).

A literatura tem demonstrado que o crescimento do uso de agrotóxicos gera impactos que podem ser percebidos na 
saúde pública (Palma, 2011; Pignati et al., 2017). Vários estudos indicam que a exposição e o consumo de agrotóxicos podem causar sintomas de intoxicação aguda e crônica à saúde humana, resultando em efeitos neurotóxicos, teratogênicos, danos ao fígado, arritmias cardíacas, alergias, asma brônquica, câncer , fibrose pulmonar, entre outros (Alavanja, Hoppin, \& Kamel, 2004; Faria, Facchini, Fassa, \& Tomasi, 2005; Curvo, Pignati, \& Pignatti, 2013; Guyton et al., 2015; Carneiro et al., 2015; Alonzo, \& Costa, 2019) e tem sido associada a episódios de suicídios (Faria, Fassa, \& Meucci, 2014).

Em outra direção, estudos apontam para uma crescente demanda de consumidores por qualidade e segurança na comercialização de alimentos vegetais frescos em todo o mundo (Aung \& Chang, 2014; Liu et al., 2018). De acordo com Andrade et al. (2013), há grande preocupação do consumidor contemporâneo com os riscos associados à contaminação de alimentos, principalmente por agrotóxicos e metais pesados, uma vez que os impactos, além de serem pouco perceptíveis, quase sempre só podem ser diagnosticados a longo prazo.

Nesse sentido, todos os envolvidos na cadeia produtiva de alimentos precisam estar atentos não só à qualidade de seus produtos, mas também às boas práticas agrícolas, no que se refere ao uso de agrotóxicos (EMBRAPA, 2004). O Estado é responsável por promover a fiscalização, o monitoramento e o controle sanitário do uso de agrotóxicos, a fim de proteger a saúde dos consumidores e garantir a preservação do meio ambiente (Brasil, 1989).

De acordo com a Lei Federal n 7.802/89, os agrotóxicos só podem ser utilizados no país após registro no órgão federal competente, a partir do atendimento as diretrizes e exigências dos órgãos da saúde, do meio ambiente e da agricultura (Brasil, 1989). O Decreto $\mathrm{n}^{\circ}$ 4.074/02 estabeleceu as competências dos três órgãos públicos envolvidos no processo de registro: Ministério da Saúde (MS), por meio da Agência Nacional de Vigilância Sanitária (Anvisa), Ministério da Agricultura, Pecuária e Abastecimento (MAPA) e Ministério do Meio Ambiente (MMA), por meio do Instituto Brasileiro do Meio Ambiente e dos Recursos Naturais Renováveis (IBAMA). Entre outras deliberações, o decreto estabeleceu que cabe aos órgãos da agricultura e da saúde o monitoramento de resíduos de agrotóxicos e afins em produtos de origem vegetal (BRASIL, 2002).

O monitoramento de agrotóxicos em alimentos de origem vegetal no Brasil é feito por meio do Programa Nacional de Controle de Resíduos e Contaminantes (PNCRC) do Ministério da Agricultura e do Programa de Análise de Resíduos de Agrotóxicos em Alimentos (PARA), coordenado pela Anvisa em parceria com estados e municípios. Nesta pesquisa serão utilizados apenas os dados do PARA, criado em 2001, com o propósito de avaliar a qualidade e segurança dos alimentos consumidos pela população e fornecer subsídios para a realização de estudos toxicológicos para fins de registro de agrotóxicos (Anvisa, 2003a).

A análise dos resultados do PARA fornece informações para a tomada de ações de mitigação de riscos, principalmente decisões sobre quais agrotóxicos e produtos alimentícios devem ser alvo de novas investigações, orientando as ações de educação, controle e fiscalização dos órgãos de vigilância sanitária (Anvisa, 2016). Além disso, avaliações permanentes da presença de resíduos de agrotóxicos em alimentos podem servir para impulsionar a implementação de ações mais efetivas que visem a adoção de boas práticas agrícolas, a mitigação de riscos à saúde humana e o cumprimento de critérios de qualidade esperados para o comércio nacional e internacional (Ciscato, Gebara, \& Monteiro, 2009).

Desta forma, o objetivo do presente estudo foi descrever e avaliar os resultados das análises de resíduos de agrotóxicos nos alimentos de origem vegetal coletados no mercado varejista de Minas Gerais pelo Programa de Análise de Resíduos de Agrotóxicos em Alimentos (PARA).

\section{Materiais e Métodos}

Trata-se de uma pesquisa descritiva de natureza quantitativa (Pereira et al., 2018). As unidades amostrais que constituem o banco de dados são os laudos laboratoriais de alimentos coletados pelo PARA no estado de Minas Gerais (PARA-MG), no período de 2013 a 2017. O acesso aos laudos de análises do PARA-MG para esta pesquisa foi autorizado, 
previamente, pela Superintendência de Vigilância Sanitária da Secretaria de Estado da Saúde de Minas Gerais.

Os laudos de análises utilizados são resultantes de coletas de alimentos realizadas no comercio varejista pelos órgãos municipais e estaduais de Vigilância Sanitária. As análises foram realizadas em laboratórios credenciados pelo programa para quantificação de resíduos de agrotóxicos e seguem métodos preconizados internacionalmente como QuEChERS (Quick, Easy, Cheap, Rugged and Safe) (Anastassiades et al., 2003) e Mini-Luke modificado (General Inspectorate For Health Protection, 1996), sendo as determinações realizadas por cromatografia líquida e/ou cromatografia gasosa, acopladas a espectrometria de massas em série, conforme a necessidade. As concentrações encontradas foram expressas em mg.kg-1 e comparadas com o nível máximo de resíduos permitido para cada ingrediente ativo (Anvisa, 2019c).

O estudo levantou dados presentes nos laudos de análises como: data da coleta, tipo de cultivar, presença de agrotóxicos por cultivar, tipos de agrotóxicos encontrados, resultados conclusivos, entre outros. Os alimentos foram categorizados em: frutas; raízes, tubérculos e bulbos; cereais e leguminosas; hortaliças não folhosas; hortaliças folhosas, enquanto os agrotóxicos classificados de acordo com seu mecanismo de ação.

Foram usados para avaliação o Limite Máximo de Resíduo (LMR), que refere-se à quantidade máxima de resíduo de agrotóxico ou afim oficialmente aceita no alimento, em decorrência da aplicação adequada do agrotóxico, e; o ingrediente ativo não autorizado para a cultura (NPC), que é aquele cujo ativo não possui LMR definido para o alimento analisado ou cujo ativo está proibido para uso no Brasil, conforme índice de monografias publicado pela Anvisa (Anvisa, 2003b).

$\mathrm{O}$ tratamento dos dados consistiu em verificar a consistência do banco de dados, realizar empilhamentos e cruzamentos de dados presentes em diferentes planilhas. Posteriormente, os resultados foram analisados e comparados com os relatórios nacionais do programa e com a literatura científica.

\section{Resultados e Discussão}

Foram analisadas 602 amostras para monitoramento de resíduos de agrotóxicos em alimentos vegetais coletados pelo PARA-MG entre 2013 e 2017. Durante o ano de 2016, as coletas do programa foram suspensas em âmbito nacional, o que comprometeu o monitoramento de resíduos de agrotóxicos em todo o país. Destaca-se que o número de amostras coletadas no estado foi reduzido de 177 (2013) para 103 (2017), uma redução de 41,8\%, quando o esperado seria a ampliação do número de coletas.

Durante o período estudado, as coletas foram realizadas em somente seis municípios mineiros, sendo que em um deles foram coletadas $91,2 \%$ das amostras nos cinco anos pesquisados. Como todas as cidades estão localizadas na região metropolitana de Belo Horizonte, pode-se afirmar que a amostra incluiu apenas alimentos comercializados na região central do estado, ou seja, é pouco representativa de outras regiões do estado.

As amostras foram coletadas em 46 estabelecimentos comerciais localizados nas áreas urbanas dos municípios participantes. Porém, $81,1 \%$ das coletas ocorreram em apenas 4 estabelecimentos pertencentes a grandes redes varejistas. Assim, a amostragem também não é representativa dos alimentos comercializados em pequenos mercados varejistas e feiras de livres, onde muitas vezes são comercializados alimentos de pequenos agricultores.

Os resultados da análise mostraram que 375 (62,3\%) das amostras de alimentos estavam contaminadas por resíduos de agrotóxicos no estado. A contaminação ficou acima de 50\% em todos os anos avaliados, principalmente em 2014 (72,6\%) e 2017 (68,9\%), que apresentaram maiores taxas de contaminação em relação a 2013 (52\%) e 2015 (58,9\%). Deste modo, o índice de contaminação de alimentos aumentou ao longo do período avaliado, muito provavelmente em função da ampliação do uso de agrotóxicos nas lavouras e da possibilidade de contaminação cruzada entre as diferentes culturas durante o plantio, colheita e pós-colheita, considerando todo o trânsito percorrido pelo alimento até chegar na fase de comercialização.

De acordo com o Instituto de Pesquisa Econômica Aplicada (2019), o uso de agrotóxicos aplicadas por área cultivada 
em Minas Gerais mais do que duplicou nos últimos anos, visto que em 2001 essa taxa era de 2,9 $\mathrm{kg} / \mathrm{ha}$ e em 2016 passou a 6,6 $\mathrm{kg} / \mathrm{ha}$, um aumento de mais de $227 \%$, o que colocou o estado entre os maiores consumidores do agrotóxicos do país, atrás apenas de Mato Grosso, São Paulo, Rio Grande do Sul, Paraná e Goiás.

Entre as amostras contaminadas, 22,6\% foram insatisfatórias para resíduos de agrotóxicos em Minas Gerais (Figura 1). Também é importante considerar o aumento do número de laudos insatisfatórios entre $2013(11,30 \%)$ e 2017 (28,16\%), o que sugere um aumento do uso indiscriminado de agrotóxicos na produção de alimentos nesse período.

Figura 1. Percentual de laudos de análises satisfatórios e insatisfatórios para resíduos de agrotóxicos em alimentos coletados no PARA-MG, entre 2013 e 2017.

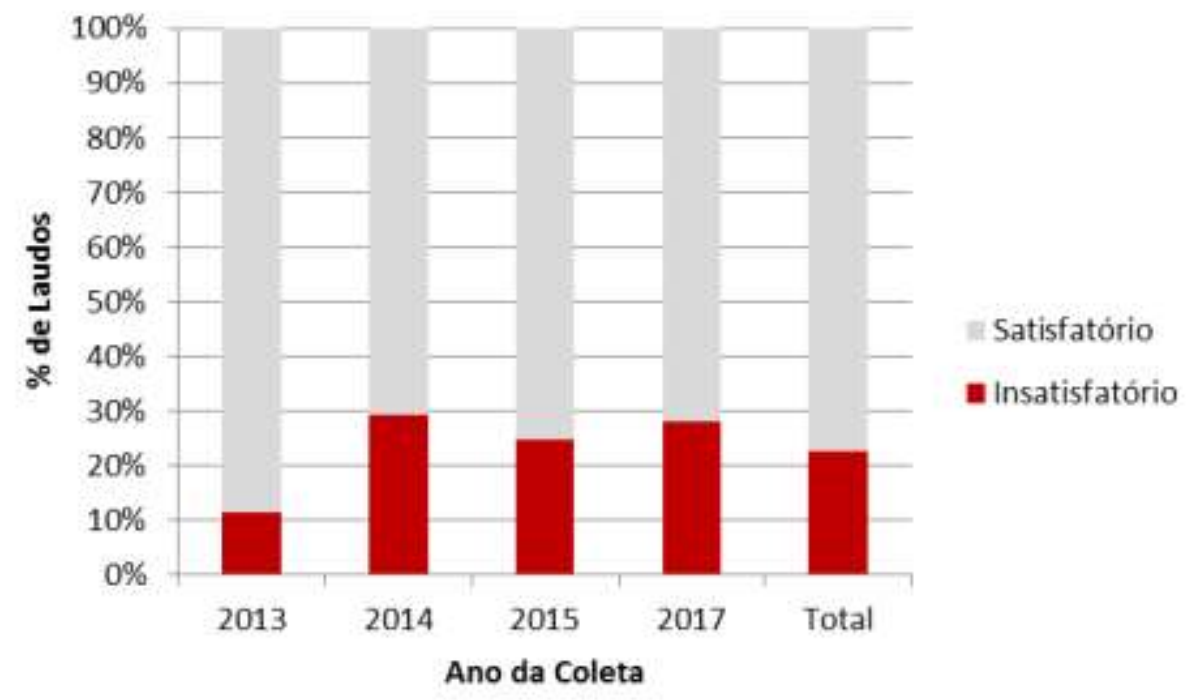

Fonte: Autores (2021).

Os resultados deste estudo indicam que praticamente $1 \mathrm{em}$ cada 4 alimentos apresentou resultado insatisfatório para agrotóxicos e que a principal causa de reprovação esteve relacionada à presença de resíduos de NPC (Tabela 1). Com exceção do percentual de amostras insatisfatórias citadas no relatório nacional do PARA em 2016, o PARA-MG apresentou percentual inferior aos publicados nos demais relatórios do programa. Entretanto, ao comparar apenas o ano de 2017 (28,16\%), os resultados no estado já se mostraram superiores aos nacionais. Cabe ressaltar que os dados do relatório de 2008 do PARA nacional não demonstrou os resultados totais de amostras insatisfatórias nas análises, apresentando apenas os resultados de algumas culturas. 
Tabela 1. Comparação dos resultados das análises das amostras do PARA-MG com os publicados em relatórios nacionais do programa.

\begin{tabular}{|c|c|c|c|c|c|c|c|}
\hline \multirow[t]{2}{*}{ Referência } & \multirow[t]{2}{*}{ Ano da coleta } & \multirow{2}{*}{$\begin{array}{c}\text { Amostras } \\
\text { analisadas } \\
(\%)\end{array}$} & \multirow{2}{*}{$\begin{array}{c}\text { Amostras } \\
\text { positivas } \\
(\%)\end{array}$} & \multicolumn{4}{|c|}{ Insatisfatórias (\%) } \\
\hline & & & & Total & $\mathrm{NPC}^{\mathrm{A}}$ & $>\mathbf{L M R}^{\mathrm{B}}$ & $\operatorname{Ambos}^{C}$ \\
\hline PARA-MG & $2013 / 2017$ & 602 & 62,3 & 22,6 & 21,6 & 3,0 & 2,0 \\
\hline Anvisa (2008) & $2001 / 2007$ & 7.321 & - & - & - & - & - \\
\hline Anvisa (2010) & $2008 / 2009$ & 3.130 & - & 29,0 & 23,8 & 2,8 & 2,4 \\
\hline Anvisa (2011) & 2010 & 2.488 & 63,0 & 27,9 & 24,3 & 1,7 & 1,9 \\
\hline Anvisa (2013) & $2011 / 2012$ & 3.062 & 42,0 & 25,0 & 21,0 & 1,9 & 1,9 \\
\hline Anvisa (2016) & $2013 / 2015$ & 12.051 & 58,0 & 19,7 & 18,3 & 3,0 & 1,7 \\
\hline Anvisa (2019) & $2017 / 2018$ & 4.616 & 51,2 & 23,2 & 20,4 & 5,4 & 2,8 \\
\hline
\end{tabular}

${ }^{A}$ Ingrediente ativo não autorizado. ${ }^{B}$ Acima do Limite Máximo de Resíduos. ${ }^{\mathrm{C}}$ Ambos os parâmetros (NPC + > LMR). Fonte: Autores (2021).

Considerando as amostras insatisfatórias, o percentual de ativos da NPC nos alimentos comercializados no estado esteve acima dos apresentados nos três últimos relatórios nacionais do programa e abaixo apenas dos percentuais dos relatórios de 2010 e 2011. O percentual de ativos acima do LMR do PARA-MG ficou abaixo do percentual divulgado no relatório nacional de 2019, idêntico ao de 2016 e superior aos anteriores. Ao comparar o percentual de reprovações em ambos os parâmetros, os resultados do PARA-MG se mostraram inferiores aos relatórios nacionais de 2010 e 2019 e superior aos demais relatórios.

O percentual de laudos insatisfatórios neste estudo foi inferior ao encontrado por Machado (2004), que avaliou 464 laudos de análises e encontrou 36\% de amostras insatisfatórias de alimentos coletados pelo PARA-MG entre 2001 e 2003, período em que as coletas do programa eram realizadas apenas na capital do estado. Deste modo é possível inferir que houve queda no número de resultados insatisfatórios para resíduos de agrotóxicos em alimentos no estado ao longo dos anos, todavia, além do menor número de alimentos, foram pesquisados somente 92 ativos entre 2001 e 2003 (Anvisa, 2008), número bastante inferior aos 273 ativos pesquisados entre 2013 e 2017.

Estudo conduzido por Amaral et al. (2012), que analisou os níveis de agrotóxicos organofosforados em 309 amostras de produtos hortícolas coletados em 44 municípios de Minas Gerais, identificou uma contaminação em 18,4\% das amostras, $17,2 \%$ dessas em desacordo com a legislação. Esses dados sugerem que a contaminação dos alimentos aumentou no estado ao longo dos anos em Minas Gerais, contudo, foi coletada praticamente a metade das amostras deste estudo e avaliados somente 27 inseticidas do grupo de organofosforados.

Os resultados deste estudo foram próximos aos encontrados no monitoramento de agrotóxicos realizado na cidade de São Paulo, que identificou 23\% de amostras insatisfatórias para agrotóxicos em alimentos analisados entre 2012 e 2016 (Tomitch et al., 2016). Por outro lado, foram menores que o encontrado no estado da Paraíba, onde foi identificado que 33\% das amostras de alimentos coletadas entre 2011 e 2013 estavam insatisfatórias (Ismael et al., 2015).

Observando apenas os resultados dos relatos insatisfatórios da Tabela 1, os dados deste estudo foram superiores aos de Machado (2004) para amostras com agrotóxicos NPC (18,7\%) e inferiores para agrotóxicos acima do LMR (17,3\%). Por outro lado, foram superiores aos de Amaral et al. (2012), que observaram 16,2\% de amostras com resíduos NPC e 1\% com resíduos acima do LMR. Esses resultados sugerem que os produtores ou desconhecem a recomendação de uso dos produtos químicos que utilizam nas lavouras ou utilizam cada vez mais agrotóxicos não autorizados nas culturas, em substituição aos 
agrotóxicos que possuem limites de uso previstos na legislação.

$\mathrm{Na}$ Tabela 2, observa-se que frutas e hortaliças não folhosas foram os grupos de alimentos com maior presença de agrotóxicos quando comparadas aos outros grupos. O mesmo ocorre para os laudos insatisfatórios, pois também apresentam maiores índices de reprovação tanto por conter ativos NPC, quanto por resíduos acima do LMR. As hortaliças folhosas não apresentaram resíduos acima do LMR. É importante ressaltar que não foram coletadas hortaliças no ano de 2017, o que impactou diretamente nos resultados deste estudo.

Tabela 2. Resultados das análises dos PARA-MG distribuídos por grupos de alimentos coletados entre os anos de 2013 e 2017.

\begin{tabular}{lccccccc}
\hline \multirow{2}{*}{ Grupos de alimentos } & $\begin{array}{c}\text { Amostras } \\
\text { analisadas }\end{array}$ & $\begin{array}{c}\text { Amostras } \\
\text { positivas (\%) }\end{array}$ & \multicolumn{4}{c}{ Amostras insatisfatórias (\%) } \\
\cline { 5 - 8 } & 226 & 85,0 & 31,0 & 28,8 & 5,8 & 3,5 \\
\hline Frutas & 132 & 35,6 & 11,4 & 11,4 & 0,8 & 0,8 \\
Raízes, tubérculos e bulbos & 126 & 53,2 & 4,0 & 3,2 & 0,8 & - \\
Cereais e Leguminosas & 69 & 82,6 & 58,0 & 58,0 & 4,3 & 4,3 \\
Hortaliças não folhosas & 49 & 24,5 & 12,2 & 12,2 & - & - \\
Hortaliças folhosas & & LMR $^{\mathbf{B}}$ & Ambos $^{\mathbf{C}}$ \\
\hline
\end{tabular}

${ }^{A}$ Ingrediente ativo não autorizado. ${ }^{B}$ Acima do Limite Máximo de Resíduos. ${ }^{\mathrm{C}}$ Ambos os parâmetros (NPC + > LMR). Fonte: Autores (2021).

As análises com resultados insatisfatórios de frutas deste estudo são superiores aos de Ciscato, Gebara, e Monteiro (2009), que a partir dos relatórios do Programa Nacional de Controle de Resíduos e Contaminantes (PNCRC) do Ministério da Agricultura, entre os anos de 2006 e 2007, evidenciaram que 23,2\% das frutas brasileiras destinadas à exportação apresentaram resultados insatisfatórios para resíduos de agrotóxicos, destas 17,8\% com presença de resíduos de NPC e 5,4\% de agrotóxicos acima do LMR, o que mostra que tanto os alimentos comercializados no país, como os exportados possuem índices preocupantes de contaminação.

Estudos como o de Hjorth et al. (2011), apontam que os países da América do Sul podem estar aumentando o uso de agrotóxicos para lucrar com a exportação de alimentos para países do norte da Europa. Esses pesquisadores demostraram que 8,4\% das frutas e hortaliças do continente americano apresentavam agrotóxicos acima do LMR e apontou o Brasil como o maior exportador desses alimentos, seguido do Uruguai e da Colômbia.

Uma pesquisa recente, que avaliou os riscos em produtos importados notificados pelos países membros da Comissão Europeia, com dados do período de 1979 a 2017, evidenciou que 8,7\% desses riscos estavam relacionados à presença irregular de resíduos de agrotóxicos em vegetais, atrás apenas das notificações relacionadas à presença de microrganismos patogênicos $(18,2 \%)$ e microtoxinas (23,0\%) (Piglowski, 2020).

Um estudo conduzido em São Paulo verificou que os teores de resíduos do agrotóxico paraquat encontrados em maçãs do tipo Fuji e Argentina, comercializadas na cidade de Mooca, não seriam aprovadas pela Comissão Europeia e pela Anvisa, por estarem acima dos limites aceitáveis (Rezende et al., 2020). Vale lembrar que o ingrediente ativo paraquate encontra-se proibido no Brasil desde 2017, devido a evidências de toxidade aguda, a associação com a Doença de Parkinson e o potencial de mutagênico deste produto (Anvisa, 2017b). Assim, a presença de resíduos de agrotóxicos nos alimentos em desacordo com a legislação nacional e internacional pode não somente comprometer a segurança dos alimentos comercializados, mas prejudicar a exportação de alimentos para outras partes do mundo.

Ao todo, foram coletadas 27 culturas de alimentos no estado de Minas Gerais pelo programa entre 2013 e 2017. 
Apesar do número desigual e irregular de amostras de alimentos coletadas entre essas culturas, os resultados insatisfatórios foram iguais ou superiores a 50\% para as amostras de abobrinha, pimentão, goiaba, uva, morango e pepino (Tabela 3).

Tabela 3. Resultados das análises de resíduos de agrotóxicos em alimentos analisados pelo PARA-MG, entre 2013 e 2017.

\begin{tabular}{|c|c|c|c|c|c|c|}
\hline \multirow[b]{2}{*}{ Alimento } & \multirow{2}{*}{$\begin{array}{l}\text { Amostras } \\
\text { analisadas }\end{array}$} & \multirow{2}{*}{$\begin{array}{c}\text { Amostras } \\
\text { positivas (\%) }\end{array}$} & \multicolumn{4}{|c|}{ Amostras insatisfatórias (\%) } \\
\hline & & & Total & NPC $^{A}$ & $>\mathbf{L M R}^{\mathrm{B}}$ & Ambos $^{C}$ \\
\hline Arroz & 49 & 34,7 & 4,1 & 2,0 & 2,0 & - \\
\hline Laranja & 40 & 100 & 17,5 & 17,5 & 2,5 & 2,5 \\
\hline Mamão & 30 & 100 & 16,7 & 16,7 & - & - \\
\hline Maçã & 30 & 100 & 13,3 & 10,0 & 3,3 & - \\
\hline Feijão & 30 & 76,7 & 6,7 & 6,7 & - & - \\
\hline Batata inglesa & 30 & 43,3 & 3,3 & 3,3 & - & - \\
\hline Tomate & 29 & 86,2 & 34,5 & 34,5 & - & - \\
\hline Fubá de milho & 29 & 41,4 & - & - & - & - \\
\hline Goiaba & 28 & 75,0 & 67,9 & 67,9 & 7,1 & 7,1 \\
\hline Cenoura & 27 & 85,2 & 33,3 & 33,3 & - & - \\
\hline Abacaxi & 22 & 81,8 & 36,4 & 31,8 & 18,2 & 13,6 \\
\hline Beterraba & 21 & 38,1 & 19,0 & 19,0 & 4,8 & 4,8 \\
\hline Pepino & 20 & 60,0 & 50,0 & 50,0 & - & - \\
\hline Banana & 20 & 20,0 & 5,0 & - & 5,0 & - \\
\hline Repolho & 20 & 20,0 & 5,0 & 5,0 & - & - \\
\hline Farinha de mandioca & 20 & - & - & - & - & - \\
\hline Morango & 19 & 100,0 & 57,9 & 57,9 & 5,3 & 5,3 \\
\hline Alface & 19 & 31,6 & 21,1 & 21,1 & - & - \\
\hline Manga & 19 & 68,4 & 15,8 & 15,8 & - & - \\
\hline Cebola & 19 & - & - & - & - & - \\
\hline Uva & 18 & 94,4 & 66,7 & 55,6 & 16,7 & 5,6 \\
\hline Farinha de trigo & 18 & 83,3 & 5,6 & 5,6 & - & - \\
\hline Abobrinha & 10 & 100,0 & 100,0 & 100,0 & 10,0 & 10,0 \\
\hline Pimentão & 10 & 100,0 & 100,0 & 100,0 & 20,0 & 20,0 \\
\hline Alho & 10 & 30,0 & 10,0 & 10,0 & - & - \\
\hline Couve & 10 & 20,0 & 10,0 & 10,0 & - & - \\
\hline Batata doce & 5 & - & - & - & - & - \\
\hline Total & 602 & 62,3 & 22,6 & 21,6 & 3,0 & 2,0 \\
\hline
\end{tabular}

${ }^{A}$ Ingrediente ativo não autorizado. ${ }^{\mathrm{B}}$ Acima do Limite Máximo de Resíduos. ${ }^{\mathrm{C}}$ Ambos os parâmetros (NPC + > LMR). Fonte: Autores (2021).

Observando os laudos insatisfatórios para as principais comodities produzidas em Minas Gerais (IBGE, 2019), os índices são mais preocupantes para morango e abacaxi e menos preocupantes para alho, feijão, banana e batata inglesa. Merece destaque que os alimentos abacaxi, morango e banana apresentaram agrotóxicos acima do LMR e que café arábica e abóbora não foram coletados no período. Considerando a importância econômica desses produtos, o PARA-MG deveria priorizar um número maior de amostras para essas culturas e incluir aquelas cuja produção também é relevante para o estado.

Os resultados da presença de resíduos de agrotóxicos NPC nos alimentos neste estudo foram superiores aos observados por Machado (2004) nas análises de morango (45,5\%), alface (6,4\%), mamão (5,5\%) e maçã (5,4\%), mas inferior para banana $(9,1 \%)$. Já quando comparados aos ativos acima do LMR, os resultados deste estudo foram superiores aos de 
Machado (2004) para: tomate $(23,5 \%)$ e maçã $(2,8 \%)$ e inferiores para batata inglesa (26,2\%). Assim, passadas quase duas décadas, os alimentos morango, alface, mamão, maçã e tomate mantiveram resultados insatisfatórios no PARA-MG, com tendência de crescimento.

Em outra pesquisa realizada em Minas Gerais, apresentaram percentuais acima de 20\% de laudos insatisfatórios as culturas de tomate, jiló e pimentão. Além disso, resíduos NPC foram identificados nas culturas de alface, cenoura, jiló, morango, pepino, pimentão e tomate e acima do LMR nas amostras de tomate (Amaral et al., 2012). Resultados que são distintos deste estudo para esses alimentos, visto que morango e pimentão foram reprovados em ambos os parâmetros, já alface, cenoura, pepino e tomate foram insatisfatórios por conter resíduos NPC.

Os vegetais com maior índice de reprovação por resíduos de agrotóxicos NPC foram, respectivamente: abobrinha, pimentão, goiaba, uva, morango e pepino. Os percentuais de reprovação por agrotóxicos NPC desta pesquisa foram superiores aos de Jardim e Caldas (2012), que avaliaram 13.556 amostras de alimentos coletadas pelos programas PARA e PNCRC em âmbito nacional, para pimentão (86,4\%), mas inferiores para: beterraba (100\%), repolho (100\%), cebola (100\%), alface (97,1\%), arroz $(93,3 \%)$, pepino $(88,4 \%)$, laranja $(86,8 \%)$, maçã $(82,2 \%)$, morango $(73,3 \%)$, couve $(71,2 \%)$, manga $(66,7 \%)$, tomate $(63,9 \%)$, uva $(62,2 \%)$, banana (52\%), feijão $(50 \%)$, abacaxi $(49,1 \%)$, cenoura $(48,2 \%)$ e mamão $(46,2 \%)$.

Entre os mais reprovados por conter agrotóxicos acima do LMR estão, respectivamente, os alimentos: pimentão, abacaxi, uva e abobrinha. No que tange os resíduos acima do LMR, os resultados foram superiores aos encontrados por Jardim e Caldas (2012) para os alimentos: abacaxi $(41,5 \%)$, uva $(21,4 \%)$, maçã $(16,8 \%)$, laranja $(13,2 \%)$ e pimentão $(2,7 \%)$, porém, foram inferiores para os alimentos: cenoura $(51,8 \%)$, feijão $(50 \%)$, mamão $(47,4 \%)$, manga $(33,3 \%)$, tomate $(27,2 \%)$, couve $(19,2 \%)$, morango $(13,5 \%)$, pepino $(7 \%)$ e alface $(1 \%)$.

Os alimentos abacaxi, pimenta, abobrinha e goiaba se destacaram tanto pela presença de resíduos de NPC, quanto acima do LMR. O percentual de reprovações em ambos os parâmetros deste estudo foi superior ao encontrado nas análises do PARA e PNCRC em nível nacional em relação aos alimentos: pimentão (10,6\%) e abacaxi $(9,1 \%)$, mas inferior para uva $(16,3 \%)$ e morango $(13,2 \%)$ (Jardim, \& Caldas, 2012).

Em comparação ao estudo de Tomitch et al. (2016), que analisaram os resultados do PARA no estado de São Paulo e encontraram resíduos de agrotóxicos acima do LMR e NPC em: abobrinha, alface, batata, beterraba, cenoura, couve, maçã, mamão, morango, pepino, pimentão, repolho, tomate e uva, a presente pesquisa do PARA em Minas Gerais identificou inadequações nos dois parâmetros em beterraba, morango, uva, abobrinha e pimentão, mas também laranja, goiaba e abacaxi. Isso demonstra a presença irregular de resíduos de agrotóxicos em alimentos comumente consumidos pela população desses dois estados.

É importante destacar que não apresentaram contaminação por resíduos de agrotóxicos os alimentos: batata-doce, cebola, farinha de mandioca e farinha de milho. A justificativa para este achado pode estar relacionada ao fato da maioria desses alimentos pertencerem ao grupo dos tubérculos raízes e bulbos, que são menos atacados por pragas e expostos aos agrotóxicos. Além disso, aqueles industrializados são submetidos à secagem para serem transformados em farinhas, o que pode promover a eliminação de resíduos.

Foram encontrados 98 agrotóxicos distintos nos alimentos analisados. Em 69,1\% das amostras constatou-se a presença de múltiplos resíduos, resultado inferior aos resultados do relatório nacional do programa de 2016, que revelaram 80\% de múltiplos resíduos (Anvisa, 2016) e superior aos resultados do relatório de 2019, que evidenciaram 34,9\% de múltiplos resíduos nas amostras (Anvisa, 2019c). Também foram superiores aos encontrados por Jardim e Caldas (2012), que apontaram 47,8\% das amostras positivas para resíduos múltiplos nas análises do PARA e PNCRC em nível nacional. No entanto, foram próximos aos verificados por Hjorth et al. (2011), que apontaram que 71\% das frutas e hortaliças importadas da América do Sul para a Europa continham múltiplos resíduos de agrotóxicos. 
Os principais alimentos que apresentaram múltiplos resíduos foram: pimentão (100\%), maçã (96,7\%), laranja (95\%), morango $(94,7 \%)$ e mamão $(93,3 \%)$. Estudos indicam que a similaridade existente no mecanismo de ação entre múltiplos resíduos nos alimentos, amplia a interação entre os mesmos e pode potencializar os efeitos adversos à saúde (Jardim \& Caldas, 2012; Ciscato \& Gebara, 2017).

Os alimentos que apresentaram maior número de resíduos na mesma amostra foram: laranja (15 resíduos), mamão (13 resíduos) e pimentão (13 resíduos). A presença de distintos resíduos em uma mesma amostra pode ser resultado da aplicação simultânea de agrotóxicos em uma mesma cultura contra diferentes pragas ou doenças, da contaminação cruzada entre lotes de alimentos tratados com diferentes agrotóxicos e da contaminação por resíduos provenientes do solo ou resultantes de derivas provenientes da pulverização aérea (EMBRAPA, 2004).

A maioria dos agrotóxicos encontrados $(85,3 \%)$ pertencia a três classes, respectivamente: fungicidas $(52,7 \%)$, acaricidas/inseticidas (17,3\%) e inseticidas (15,9\%). Segundo Ciscato e Gebara (2017), o uso frequente de fungicidas e inseticidas pode estar associado ao clima tropical brasileiro, que propicia o aumento do ataque de diversos tipos de fungos e insetos às plantações.

Os agrotóxicos mais comumente encontrados nos alimentos foram carbendazim $(11,4 \%)$, ditiocarbamatos $(6,9 \%)$ e acefato $(5,3 \%)$. Resultados semelhantes aos publicados no relatório nacional do programa em 2016 (Anvisa, 2016), que evidenciaram em maior quantidade: carbendazim $(11,2 \%)$, ditiocarbamato $(6,7 \%)$ e acefato $(5,6 \%)$. Porém, foram diferentes do último relatório do programa, que apontou maior quantidade de ativos: imidacloprido (15,5\%), tebuconazol (12,5\%) e carbendazim (11,4\%) (Anvisa, 2019c). Todavia, embora o carbendazim seja autorizado no Brasil, seu uso está proibido nos países da União Europeia (ESFA, 2010) e limitado nos Estados Unidos para uso não agrícola (USEPA, 2014), assim como o acefato não está autorizado nos países da União Europeia (IPEA, 2019).

Um estudo realizado na região da Lombardia, na Itália, demonstrou que a contaminação e a presença de múltiplos resíduos em alimentos vegetais convencionais chegam a ser 10 vezes maiores do que os encontrados em alimentos orgânicos (Tasiopoulou et al., 2007). Assim, embora não seja conhecido o local exato de produção dos alimentos coletados pelo PARAMG, tanto a origem quanto as formas de cultivo precisam fazer parte dos dados deste programa, a fim de mensurar o risco associado às diferentes práticas agrícolas e identificar os produtores que não atendem aos regulamentos nacionais que limitam a aplicação de agrotóxicos.

De acordo com Cao, et al. (2017), uma das formas de minimizar os riscos à segurança dos alimentos seria por meio do rastreamento da fonte desses perigos, a partir do monitoramento do fluxo dos produtos alimentares. Ribeiro et al. (2020) apontaram que o desenvolvimento de tecnologias de rastreabilidade, apoiadas especialmente no uso da internet e da inteligência artificial, se apresenta como um importante reforço na garantia de precisão e fluxo seguro de informações na cadeia produtiva de alimentos.

Os achados do PARA-MG, quanto aos tipos de agrotóxicos encontrados, não correspondem aos dados de comercialização de agrotóxicos disponibilizados pelo Ibama (IBAMA, 2017), que apontou o glifosato (173,15 t), 2,4-D (57,39 t) e maconzeb $(30,15$ t), respectivamente, como os produtos mais comercializados no país em 2017. Apenas o glifosato respondeu por mais da metade das vendas de agrotóxicos naquele ano. Ademais, o fato do glifosato e do herbicida 2,4-D terem sido incluídos no programa apenas em 2017, justifica os menores percentuais encontrados nos alimentos em relação aos demais (Anvisa, 2016; Friedrich, Souza, \& Carneiro, 2018).

Os resultados deste estudo também divergem dos de Jardim e Caldas (2012), que encontraram em maior quantidade os agrotóxicos: diocarbamatos $(41,6 \%)$, carbendazim $(26,7 \%)$ e clorpirifós $(16,1 \%)$ na análise dos programas nacionais, PARA e PNCRC. E no estudo de Ciscato, Gebara e Monteiro (2009), os principais agrotóxicos encontrados em frutas coletadas no PNCRC foram: ditiocarbamatos $(23,1 \%)$ e piretróides $(21,8 \%)$. 
Considerando os resultados apresentados na Tabela 4, os agrotóxicos carbendazim, imidacloprido, diocarbamatos, acefato e etefrom foram detectados com maior frequência nos laudos e juntos totalizam 66,7\% dos resíduos acima do LMR nos alimentos analisados. É importante ressaltar que resíduos de carbofurano foram encontrados acima do limite em uma amostra de laranja (5,6\%), apesar do uso ser proibido no Brasil (Anvisa, 2017a).

Tabela 4. Resíduos de agrotóxicos acima do LMR encontrados nos alimentos monitorados no PARA-MG, entre 2013 e 2017.

\begin{tabular}{|c|c|c|c|}
\hline Agrotóxicos & $\begin{array}{c}\text { Classe } \\
\text { (Grupo Químico) }\end{array}$ & $\begin{array}{c}\text { Ensaios } \\
\text { Insatisfatórios } \\
(\%)\end{array}$ & $\begin{array}{l}\text { Alimentos positivos } \\
\text { (n. }{ }^{\circ} \text { de amostras) }\end{array}$ \\
\hline Carbendazim & $\begin{array}{c}\text { Fungicida } \\
\text { (Benzimidazol) }\end{array}$ & $4(22,2 \%)$ & Abacaxi (4) \\
\hline Imidacloprido & $\begin{array}{c}\text { Inseticida } \\
\text { (Neonicotinóide) }\end{array}$ & $2(11,1 \%)$ & Goiaba (1), Abacaxi (1) \\
\hline Ditiocarbamatos & $\begin{array}{c}\text { Fungicida } \\
\text { (Alquilenobis) }\end{array}$ & $2(11,1 \%)$ & Morango (1), Beterraba (1) \\
\hline Acefato & $\begin{array}{l}\text { Acaricida/Inseticida } \\
\text { (Organofosforado) }\end{array}$ & $2(11,1 \%)$ & Pimentão (2) \\
\hline Etefom & $\begin{array}{l}\text { Regulador de Crescimento } \\
\text { (Etileno - precursor de) }\end{array}$ & $2(11,1 \%)$ & Uva (2) \\
\hline Tebuconazol & $\begin{array}{l}\text { Fungicida } \\
\text { (Triazol) }\end{array}$ & $1(5,6 \%)$ & Banana (1) \\
\hline Tiametoxam & $\begin{array}{c}\text { Inseticida } \\
\text { (Neonicotinóide) }\end{array}$ & $1(5,6 \%)$ & Abobrinha (1) \\
\hline Cipermetrina & $\begin{array}{l}\text { Inseticida } \\
\text { (Piretróide) }\end{array}$ & $1(5,6 \%)$ & Arroz (1) \\
\hline Carbofurano* & $\begin{array}{c}\text { Inseticida } \\
\text { (Metilcarbamato de benzofuranila) }\end{array}$ & $1(5,6 \%)$ & Laranja (1) \\
\hline Pirimetanil & $\begin{array}{c}\text { Fungicida } \\
\text { (Anilinopirimidina) }\end{array}$ & $1(5,6 \%)$ & Maçã (1) \\
\hline Indoxabarbe & $\begin{array}{l}\text { Cupinicida/formicida } \\
\text { (Oxadiazina) }\end{array}$ & $1(5,6 \%)$ & Uva (1) \\
\hline
\end{tabular}

*Uso proibido no Brasil até o ano de 2017 (Anvisa, 2017a). Fonte: Autores (2021).

A Tabela 5 apresenta os principais resíduos de agrotóxicos NPC presentes nos alimentos pesquisados. Entre eles estão: acefato, metamidófos, acetamiprido, clorpirifós e carbendazim, que juntos representam 45,8\% dos ativos detectados irregularmente. Dentre esses agrotóxicos, dois são proibidos pela legislação brasileira para uso em alimentos: metamidófos e metalaxil (Anvisa, 2019d). Embora com menor frequência, o estudo revelou a presença de outros agrotóxicos NPC: aldicarbe sulfóxido $(0,4 \%)$, fentoato $(1,6 \%)$, hexaconazol $(0,4 \%)$ e ometoato $(0,8 \%)$ em amostras de goiaba; dicofol $(0,8 \%)$ em laranja; parationa-metilica $(0,8 \%)$ em morango; procloraz $(0,4 \%)$ em mamão, e; protiofos (tokution) $(0,8 \%)$ em amostras de arroz e beterraba. 
Tabela 5. Resíduos de agrotóxicos NPC encontrados em alimentos monitorados no PARA-MG, entre 2013 e 2017.

\begin{tabular}{|c|c|c|c|}
\hline Agrotóxicos & $\begin{array}{c}\text { Classe } \\
\text { (Grupo Químico) }\end{array}$ & $\begin{array}{c}\text { Ensaios } \\
\text { Insatisfatórios (\%) }\end{array}$ & $\begin{array}{l}\text { Alimentos positivos } \\
\text { (n. }{ }^{\circ} \text { de amostras) }\end{array}$ \\
\hline Acefato & $\begin{array}{l}\text { Acaricida/Inseticida } \\
\text { (Organofosforado) }\end{array}$ & $34(13,7 \%)$ & $\begin{array}{l}\text { Pepino (7), goiaba (6), uva (6), abobrinha (4), } \\
\text { cenoura (3), morango (3), tomate (3), } \\
\text { beterraba (1), manga (1). }\end{array}$ \\
\hline Metamidófós* & $\begin{array}{l}\text { Acaricida/Inseticida } \\
\text { (Organofosforado) }\end{array}$ & $28(11,2 \%)$ & $\begin{array}{l}\text { Pimentão (8), pepino (6), tomate (6), } \\
\text { morango (3), cenoura (2), abobrinha (2), } \\
\text { repolho (1), uva (1). }\end{array}$ \\
\hline Acetamiprido & $\begin{array}{l}\text { Inseticida } \\
\text { (Neonicotinóide) }\end{array}$ & $20(8,0 \%)$ & $\begin{array}{c}\text { Pimentão (7), abobrinha (7), pepino (3), uva } \\
\text { (2), morango (1). }\end{array}$ \\
\hline Clorpirifós & $\begin{array}{l}\text { Acaricida/Formicida/Inse } \\
\text { ticida (Organofosforado) }\end{array}$ & $17(6,8 \%)$ & $\begin{array}{c}\text { Tomate (7), abobrinha (4), cenoura (2), alface } \\
\text { (2), couve (1), morango (1). }\end{array}$ \\
\hline Carbendazim & $\begin{array}{c}\text { Fungicida } \\
\text { (Benzimidazol) }\end{array}$ & $15(6 \%)$ & Abobrinha (7), pimentão (5), goiaba (3). \\
\hline Benalaxil & $\begin{array}{c}\text { Fungicida } \\
\text { (Acilalaninato) }\end{array}$ & $7(2,8 \%)$ & Abobrinha (4), pepino (2), pimentão (1). \\
\hline Metalaxil* & $\begin{array}{l}\text { Fungicida } \\
\text { (Acilalaninato) }\end{array}$ & $6(2,4 \%)$ & $\begin{array}{l}\text { Pimentão (3), morango (1), pepino (1), uva } \\
\text { (1). }\end{array}$ \\
\hline Fluazifope-p-butil & $\begin{array}{l}\text { Herbicida (Ácido } \\
\text { ariloxifenoxipropiônico) }\end{array}$ & $6(2,4 \%)$ & Goiaba (5), alho (1). \\
\hline Metomil & $\begin{array}{l}\text { Acaricida/Inseticida } \\
\text { (Metilcarbamato de } \\
\text { oxima) }\end{array}$ & $6(2,4 \%)$ & $\begin{array}{c}\text { Pimentão (3), pepino (1), morango (1), uva } \\
\text { (1). }\end{array}$ \\
\hline
\end{tabular}

*Uso proibido no Brasil (Anvisa, 2019d). Fonte: Autores (2021).

De modo geral, 5,8\% das amostras analisadas pelo programa em Minas Gerais estavam contaminadas com agrotóxicos proibidos, número seis vezes superior ao último relatório nacional do PARA (Anvisa, 2019c), que mostrou apenas 0,9\% das amostras com ativos proibidos. Os maiores percentuais de reprovação para resíduos de agrotóxicos proibidos foram para as frutas: goiaba $(32,1 \%)$ e morango $(21,1 \%)$ e para as hortaliças não folhosas: pimentão $(60 \%)$, pepino $(40 \%)$, abobrinha $(40 \%)$ e tomate $(24,2 \%)$. Vale ressaltar que os ativos proibidos encontrados nesta pesquisa foram responsáveis por $22,59 \%$ das detecções de resíduos de NPC.

Esses resultados apontam para o uso de agrotóxicos de forma indiscriminada e às vezes ilegal em culturas para quais não são autorizados ou permitidos, situação que pode se apresentar como um fator de risco para o trabalhador rural, para o consumidor e para o meio ambiente. Considerando à toxicidade de alguns produtos, o uso inadequado pode levar a exposição direta ou a ingestão de alimentos contaminados, bem como resultar no lançamento desses produtos e suas embalagens no solo ou na água para consumo humano.

A dificuldade de se conhecer a origem da contaminação é um dos fatores que limitam o controle sobre o uso de agrotóxicos nas plantações. Embora a implementação da rastreabilidade ao longo da cadeia produtiva de produtos vegetais frescos destinados à alimentação humana se tornou obrigatória no território brasileiro (Brasil, 2018), a maioria das tecnologias de rastreabilidade disponíveis estão associadas principalmente à logística, em detrimento a aspectos relacionados à segurança 
alimentar e ao controle sanitário sobre resíduos de agrotóxicos em alimentos vegetais (Ribeiro et al., 2020).

Apesar da existência de previsão nas normas para destruição ou inutilização de vegetais ou parte desses sempre que apresentarem resíduos de agrotóxicos acima dos níveis permitidos ou quando tenha havido aplicação de agrotóxicos e afins de uso não autorizado no Brasil (Brasil, 2002), as diretrizes do PARA não estabelecem a realização de medidas de natureza fiscal, o que se traduz em insegurança quanto a efetividade do controle sobre esses resíduos nos alimentos. Contudo, a competência de legislar e fiscalizar o uso, a produção, o consumo, o comércio e o armazenamento de agrotóxicos foi delegada aos estados e ao distrito federal na legislação brasileira, que também estabeleceu penalidades de natureza administrativa, civil e penal em casos envolvendo danos à saúde das pessoas e ao meio ambiente (Brasil, 1989).

É importante destacar a baixa escolaridade entre os agricultores que usam agrotóxicos em Minas Gerais, uma vez que cerca de 34\% deles possuem apenas o ensino fundamental e 7\% se declaram analfabetos. Além disso, 52,9\% relatam não ter recebido nenhuma assistência técnica para o uso de agrotóxicos (IBGE, 2019).

Recentemente, foi instituída a Política Estadual de Agroecologia e Produção Orgânica em Minas Gerais (Minas Gerais, 2014) e o Plano de Ação da Estratégia Intersetorial de Redução do Uso de Agrotóxicos e Apoio à Agroecologia e à Produção Orgânica no estado (Minas Gerais, 2018). Além disso, o governo estadual instituiu o Programa Mineiro de Monitoramento da Qualidade dos Produtos e Serviços Sujeitos ao Controle Sanitário (PMQPS), o qual passou a incorporar as ações do PARA em 2019 (Minas Gerais, 2019). Com isso, o estado criou condições de implementar medidas para desencorajar o uso de agrotóxicos, valorizar e certificar a produção orgânica e agroecológica, promover às boas práticas agrícolas, ampliar o monitoramento de resíduos de agrotóxicos nos alimentos comercializados e fomentar o uso de sistemas da rastreabilidade, assim como de executar ações de natureza regulatória, fiscalizatória e educativa sobre a cadeia produtiva de alimentos.

\section{Conclusão}

Os resultados deste estudo permitiram evidenciar a existência de contaminação por resíduos de agrotóxicos na maioria dos alimentos de origem vegetal coletados pelo PARA no comércio varejista em Minas Gerais entre os anos de 2013 e 2017. Cerca de 1/4 dos alimentos apresentaram irregularidades perante a legislação sanitária, sendo que o maior número de reprovações esteve relacionado à presença de resíduos de agrotóxicos não autorizados para as culturas, muitos dos quais são de uso proibido no Brasil.

Os resultados encontrados no estado estiveram muito próximos aos divulgados no último relatório nacional do programa. As hortaliças não folhosas e as frutas foram as categorias com maior número de reprovações, tanto por conterem agrotóxicos não autorizados para a cultura, como acima dos limites máximos de resíduos permitidos pela legislação brasileira.

Os resíduos de agrotóxicos mais presentes nos alimentos comercializados no estado foram dos ativos: carbendazim, ditiocarbamatos e acefato. Entretanto, o programa não analisou a presença de glifosato, 2.4-D e maconzeb no período anterior a 2017, que estão entre os agrotóxicos mais comercializados no país.

Os critérios de amostragem utilizados pelo PARA não são claros e a descontinuidade no monitoramento de algumas culturas dificultam a definição de uma série histórica de análises de alguns alimentos no estado. Todavia, a presença de agrotóxicos em desacordo com a legislação brasileira e a persistência de resultados insatisfatórios ao longo dos anos pesquisados, sugerem falhas nas boas práticas agrícolas e apontam para a importância de ações eficazes de controle e mitigação dos riscos frente a contaminação de alimentos por agrotóxicos.

Pesquisas futuras podem ser dirigidas ao estudo das fragilidades envolvidas na execução do PARA e na atuação dos órgãos de controle que propiciam a manutenção de resultados insatisfatórios ao longo do tempo, bem como para a avaliação dos riscos associados ao uso de agrotóxicos para a saúde e para a biodiversidade. 


\section{Agradecimentos}

Os autores agradecem à Superintendência de Vigilância Sanitária da Secretaria de Estado da Saúde de Minas Gerais pelo acesso aos laudos do programa e ao Laboratório de Estatística do Departamento de Estatística da Universidade Federal de Minas Gerais pelo auxílio nas análises dos dados.

\section{Referências}

Agência Nacional de Vigilância Sanitária. (2003a). Resolução da Diretoria Colegiada. RDC n. ${ }^{\circ} 119$, de 19 de maio de 2003 . Diário Oficial da União n. 97 Seção 1, p 39 .

Agência Nacional de Vigilância Sanitária. (2003b). Resolução da Diretoria Colegiada. RDC n. ${ }^{\text {o }} 165$, de 29 de agosto de 2003. Diário Oficial da União, Brasília, DF, 2 de setembro. p. 48.

Agência Nacional de Vigilância Sanitária. (2008). Programa de Análise de Resíduos de Agrotóxicos (PARA): relatório de atividades 2001 A 2007. Brasília: Anvisa. http://portal.anvisa.gov.br/programa-de-analise-de-registro-de-agrotoxicos-para

Agência Nacional de Vigilância Sanitária. (2010). Programa de Análise de Resíduos de Agrotóxicos (PARA): relatório de atividades 2009. Brasília: Anvisa. http://portal.anvisa.gov.br/programa-de-analise-de-registro-de-agrotoxicos-para

Agência Nacional de Vigilância Sanitária. (2011). Programa de Análise de Resíduos de Agrotóxicos (PARA): relatório de atividades 2010. Brasília: Anvisa. http://portal.anvisa.gov.br/programa-de-analise-de-registro-de-agrotoxicos-para

Agência Nacional de Vigilância Sanitária. (2012). Seminário volta a discutir mercado de agrotóxicos em 2012. Imprensa/Anvisa. http://portal.anvisa.gov.br/

Agência Nacional de Vigilância Sanitária. (2013). Programa de Análise de Resíduos de Agrotóxicos (PARA): relatório de atividades 2011 a 2012. Brasília: Anvisa. http://portal.anvisa.gov.br/programa-de-analise-de-registro-de-agrotoxicos-para

Agência Nacional de Vigilância Sanitária. (2016). Programa de Análise de Resíduos de Agrotóxicos (PARA): relatório das análises de amostras monitoradas no período de 2013 a 2015. Brasília: Anvisa. http://portal.anvisa.gov.br/programa-de-analise-de-registro-de-agrotoxicos-para

Agência Nacional de Vigilância Sanitária (2017a). Resolução nº 185, de 18 de outubro de 2017. Diário Oficial da União, Brasília, DF, 19 de outubro de 2017. Seção 1. p. 32.

Agência Nacional de Vigilância Sanitária. (2017b), Resolução RDC № 190, de 30 de novembro de 2017. Altera a resolução da diretoria colegiada N 177, de 21 de setembro de 2017. Diário Oficial União. 1 dez 2017.

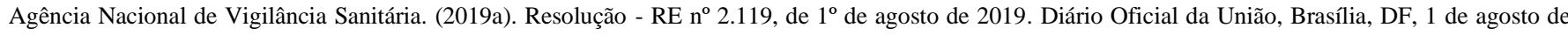
2019. p. 56.

Agência Nacional de Vigilância Sanitária. (2019b). Resolução da Diretoria Colegiada - RDC no 294, de 29 de julho de 2019. Diário Oficial da União, Brasília, DF, 11 de julho de 2019. Seção 1. p. 56.

Agência Nacional de Vigilância Sanitária. (2019c). Programa de Análise de Resíduos de Agrotóxicos (PARA): relatório de atividades 2017 a 2018. Brasília: Anvisa http://portal.anvisa.gov.br/programa-de-analise-de-registro-de-agrotoxicos-para

Agência Nacional de Vigilância Sanitária (2019d). Listas de ingredientes ativos com uso autorizado e banidos no Brasil. https://www.gov.br/anvisa/ptbr/assuntos/noticias-anvisa/2017/listas-de-ingredientes-ativos-com-uso-autorizado-e-banidos-no-brasil

Alavanja, M. C. R., Hoppin, J. A., \& Kamel, F. (2004). Health effects of chronic pesticide exposure: cancer and neurotoxicity. Annu. Rev. Public Health, 25(1), 155-197. https://doi:10.1146/annurev.publhealth.25.101802.123020

Alonzo, H. G. A., \& Costa, A. O. (2019). Bases da Toxicologia ambiental e clínica para atenção à saúde: exposição e intoxicação por agrotóxicos. São Paulo: Hucitec.

Amaral, E. H., Soares, A. A, Sousa, L. A. F, Souza, S. V. C, \& Junqueira, R. G. (2012). Resíduos de inseticidas organofosforados: validação de método e ocorrência em hortícolas. Rev Inst Adolfo Lutz, 71(2). 345-54.

Anastassiades, M., Lehotay, S. J., Stajnbaher, D., \& Schenck, F. L. (2003). Fast and Easy Multiresidue Method Employing Acetonitrile Extraction/Partitioning and "Dispersive Solid-Phase Extraction" for the determination of Pesticides Residues in Produce. J. AOAC Int, 86(2), $412-431$.

Andrade, J. C., Seliza, R., Yamada, E. A., Galvão, M. T. E. L., Frewer, L. J., \& Beraquet, N. J. (2013). Percepção do consumidor frente aos riscos associados aos alimentos, sua segurança e rastreabilidade. Braz. J. Food Technol, 16(3), 184-191. http://dx.doi.org/10.1590/S1981-67232013005000023

Aung, M. M., \& Chang, Y. S. (2014). Traceability in a food supply chain: Safety and quality perspectives. Food Control, 39, 172-184. https://doi: 10.1016/j.foodcont.2013.11.007

Balsan, R. (2006). Decurrent impacts of the agriculture modernization in Brazil. Campo-Território: Revista de Geografia Agrária, 1, 123 -151.

Bombardi, L. M. (2017). Geografia do uso de agrotóxicos no Brasil e conexões com a União Europeia. São Paulo: FFLCH - USP https://www.larissabombardi.blog.br/atlas2017

Brasil. (1989). Lei no 7.802, de 11 de julho de 1989 da. Diário Oficial da União, Brasília, DF, 12 de julho de 1989, Seção 1. 11459. 
Brasil. (2002). Decreto n o 4.074, de 4 de janeiro de 2002. Diário Oficial da União, Brasília, DF, 8 de janeiro de 2002, n. ${ }^{\circ}$ 5, Seção 1. 1.

Brasil. (2018). Ministério da Agricultura, Pecuária e Abastecimento; Agência Nacional de Vigilância Sanitária. Instrução normativa conjunta n. ${ }^{\circ} 02$, de 7 de fevereiro de 2018. Diário Oficial da União, Brasília, DF, 8 fev. Seção 1, p. 148-149.

Brasil. (2021). Ministério da Agricultura, Pecuária e Abastecimento. AGROFIT: Sistema de Agrotóxicos Fitossanitários. 2021. www.agricultura.gov.br/servicos-e-sistemas/sistemas/agrofit

Cao, Y., Liu, X., Guan, C., \& Mao, B. (2017). Implementation and Current Status of Food Traceability System in Jiangsu China. Procedia Computer Science, 122, 617-621. https://doi:10.1016/j.procs.2017.11.414

Carneiro, F. F., Augusto, L. G. S., Rigotto, R. M., Friedrich, K., \& Búrigo, A. C. (2015). Dossiê ABRASCO: um alerta sobre os impactos dos agrotóxicos na saúde. São Paulo: Expressão Popular. http://abrasco.org.br/dossieagrotoxicos/

Ciscato, C. H. P., \& Gebara, A. B. (2017). Avaliação de resíduos de pesticidas na dieta brasileira, período de 2001 a 2010. Higiene Alimentar, 31(274/275), $110-114$.

Ciscato, C. H. P., Gebara, A. B., \& Monteiro, S. H. (2009). Pesticide residue monitoring of Brazilian fruit for export 2006-2007. Food Additives \& Contaminants: Part B. 2009, 2(2), 140-145. https://doi: 10.1080/19440040903330326

Curvo, H. R. M., Pignati, W. A., \& Pignatti, M. G. (2013). Morbimortalidade por câncer infanto-juvenil associada ao uso agrícola de agrotóxicos no Estado de Mato Grosso, Brasil. Cad. Saúde Colet, 21(1), 10-17. https://doi.org/10.1590/S1414-462X2013000100003

EFSA. European Food Safety Authority. (2010). Conclusion on Pesticide Peer Review. Conclusion on the Peer Review of th Pesticide Risk Assessment of the Active Substance

Carbendazim. EFSA Journal, 8(5), 1-76. https://efsa.onlinelibrary.wiley.com/doi/epdf/10.2903/j.efsa.2010.1598

Empresa Brasileira de Pesquisa Agropecuária. (2004). Manual de boas práticas agrícolas e sistema APPCC. Brasília: EMBRAPA. https://ainfo.cnptia.embrapa.br/digital/bitstream/item/18226/1/MANUALBOASPRATICASAGRICappcc.pdf

Faria, N. M. X., Facchini, L. A., Fassa, A. G., \& Tomasi, E. (2005). Pesticides and respiratory symptoms among farmers. Rev Saúde Pública, $39(6), 973-981$. https://doi.org/10.1590/S0034-89102005000600016

Faria, N. M. X., Fassa, A. G. F., \& Meucci, R. D. (2014). Association between pesticide exposure and suicide rates in Brazil. Neurotoxicology, 45, 355-362. https://doi: 10.1016/j.neuro.2014.05.003

Friedrich, K.; Souza, M. M. O., \& Carneiro, F. F. (2018). Dossiê científico e técnico contra o Projeto de Lei do Veneno (PL 6.299/2002) e a favor do Projeto de Lei que institui a Política Nacional de Redução de Agrotóxicos - PNARA. Rio de Janeiro: ABRASCO/ABA.

General Inspectorate for Health Protection. (1996). Analytical Methods for Pesticide Residues in Foodstuffs. (6a ed.). The Netherlands: Ministry of Public Health, Welfare and Sports.

Guyton, K. Z., Loomis, D., Grosse, Y., El Ghissassi, F., Benbrahim-Tallaa, L., Guha, N., \& Straif, K. (2015). Carcinogenicidade de tetraclorvinfos, paration, malation, diazinon e glyphosate. The Lancet Oncology, 16(5), 490-491. https://doi: 10.1016/S1470-2045(15)70134-8

Hjorth, K., Johansen, K., Holen, B., Andersson, A., Christensen, H. B., Siivinen, K., \& Toome, M. (2011). Pesticide residues in fruits and vegetables from South America e A Nordic project. Food Control, 22(11), 1701-1706. https://doi: 10.1016/j.foodcont.2010.05.017

Instituto Brasileiro de Geografia e Estatística. (2019). Censo agropecuário: resultados definitivos 2017. Rio de Janeiro: IBGE. Recovered from https://censos.ibge.gov.br/agro/2017/resultados-censo-agro-2017.html

Instituto Brasileiro do Meio Ambiente e dos Recursos Naturais Renováveis. (2017). Relatórios de comercialização de agrotóxicos. Boletim 2017. Brasília: IBAMA. http://www.ibama.gov.br/agrotoxicos/relatorios-de-comercializacao-de-agrotoxicos\#boletinsanuais

Instituto de Pesquisa Econômica Aplicada. (2019). Agrotóxicos no Brasil: padrões de uso, política da regulação e prevenção da captura regulatória texto para discussão. Brasília: IPEA, 76 p. http://repositorio.ipea.gov.br/bitstream/11058/9371/1/td_2506.pdf

Ismael, L.L., Rocha, E.M.R., Lins Filho, L.A., \& Lima, R. P. A. (2015). Resíduos de agrotóxicos em alimentos: preocupação ambiental e de saúde para população paraibana. Revista Verde de Agroecologia e Desenvolvimento Sustentável, 10(3), 24-29. http://dx.doi.org/10.18378/rvads.v10i3.3459

Jardim, A. N. O., \& Caldas, E. D. (2012). Brazilian monitoring programs for pesticide residues in food e Results from 2001 to 2010. Food Control, 25(2), 607616. https://doi.org/10.1016/j.foodcont.2011.11.001

Liu, C., Li, J., Steele, W., \& Fang, X. (2018). A study on Chinese consumer preferences for food traceability information using best-worst scaling. PLoS ONE, 13(11), 1-16. https://doi.org/10.1371/journal.pone.0206793

Machado, C. P. (2004). Estimativa do risco da ingestão de resíduos de agrotóxicos em hortifrutícolas comercializadas em Belo Horizonte [master's thesis]. Belo Horizonte: Faculdade de Farmácia, Universidade Federal de Minas Gerais.

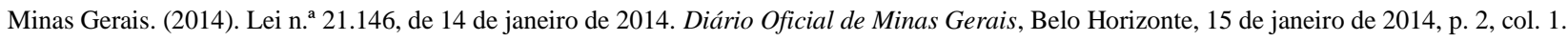

Minas Gerais. (2018). Decreto n. ${ }^{\circ}$ 481, de 25 de setembro de 2018. Diário Oficial de Minas Gerais, Belo Horizonte. 26 de setembro de 2018, p. 2 , col. 2.

Minas Gerais. (2019). Resolução SES/MG no 6.711, de 17 de abril de 2019. Diário Oficial de Minas Gerais, Belo Horizonte, 17 de abril de 2019, Seção 1. p 22. 
Neto, E. M., Lacaz, F. A. C., \& Pignati, W. A. (2014). Vigilância em saúde e agronegócio: os impactos dos agrotóxicos na saúde e no ambiente. Perigo à vista! Ciência \& Saúde Coletiva, 19(12), 4709-4718. http://dx.doi.org/10.1590/1413-812320141912.03172013

Palma, D. C. A. (2011). Agrotóxicos em leite humano de mães residentes em Lucas do Rio Verde [master’s thesis]. Cuiabá: Universidade Federal de Mato Grosso.

Pelaez, V. M., Silva, L. R. da, Guimarães, T. A., Ri, F. D., \& Teodorovicz, T. (2015). A (des)coordenação de políticas para a indústria de agrotóxicos no Brasil. Rev. Bras. Inov, 14, 153-178. https://doi.org/10.20396/rbi.v14i0.8649104

Pereira, A. S., Shitsuka, D. M., Parreira, F. J., Shitsuka, R. (2018). Metodologia da pesquisa científica. 1. ed. Santa Maria: Universidade Federal de Santa Maria.

Pigłowski, M. (2020). Food hazards on the European Union market: The data analysis of the Rapid Alert System for Food and Feed. Food Sci Nutr. 2020, 125. https://doi: 10.1002/fsn3.1448

Pignati, W. A., Lima, F. A. N. de S., Lara, S. S. de, Correa, M. L. M., Barbosa, J. R., Leão, L. H. da C., \& Pignatti, M. G. (2017). Distribuição espacial do uso de agrotóxicos no Brasil: uma ferramenta para a Vigilância em Saúde. Ciência e Saúde Coletiva, 22(10), 3281-3293. http://dx.doi.org/10.1590/1413812320172210.17742017

Rezende, R. A. E. de, Nicoletti, M. A., Pereira, A. G. A., Serafim, B. O., Paixão, D. F., Diniz, J. S., Gama, L. C. B., Silva, L. P., Munoz, J. W. P., \& Fukushima, A. R. (2020). Determinação de Paraquat em maçãs do tipo Gala, Fuji, Argentina e Verde. Vigil. sanit. debate. 8(1), 106-110. https://doi.org/10.22239/2317-269x.01341

Ribeiro, M. C., Ramos, A. M., Ferreira, V. A., Cunha, J. R. da, \& Fante, C. A. (2020). Tecnologias de rastreabilidade, segurança e controle de resíduos de agrotóxicos na cadeia produtiva de alimentos de origem vegetal: um estudo de revisã0. Research, Society and Development, 9(12), e5291210780. DOI: 10.33448/rsd-v9i12.10780.

Santos M., \& Glass, V. (2018). Altas do agronegócio: fatos e números sobre as corporações que controlam o que comemos. Rio de Janeiro: Fundação Heinrich Böll. https://br.boell.org/sites/default/files/atlas_agro_final_06-09.pdf

Schreinemachers, P., \& Tipraqsa, P. (2012) Agricultural pesticides and land use intensification in high, middle and low income countries. Food Policy, 37(6), 616-626. https://doi: 10.1016/j.foodpol.2012.06.003

Tasiopoulou, S., Chiodini, A. M., Vellere, F., \& Visentin, S. (2007). Results of the monitoring program of pesticide residues in organic food of plant origin in Lombardy (Italy). Journal of Environmental Science and Health, Part B, 42(7), 835-841. https://doi.org/10.1080/03601230701555054

Tomitch, R., Barchi, C. W. C. B., Feitosa, M. C. J. C. de, Vaz Lobo, R. S., \& Tamega Junior, W.P. (2016). Analysis program of pesticide residues in food PARA: Tracking food with unsatisfactory results in São Paulo. In Anais...XXX Congresso de Secretários Municipais de Saúde do Estado de São Paulo. São Paulo. 2016. https://pesquisa.bvsalud.org/sms/resource/pt/sms-11938

United States Environmental Protection Agency. (2014). Thiophanate-Methyl and Carbendazim (MBC). Human Health Assessment Scoping Document in Support of Registration Revies. Memorandum. EUA: USEPA. https://archive.epa.gov/pesticides/reregistration/web/pdf/tm_red.pdf 